Classification

Physics Abstracts

$61.16 \mathrm{Ch}-81.60 \mathrm{Bn}-68.35 \mathrm{Fx}-73.40 \mathrm{Gk}$

\title{
Nano-écriture sur couche d'or
}

\author{
Chrystel Lebreton et Zhao Zhong Wang \\ Laboratoire de Microélectronique et Microstructures (L2M) / CNRS, 196 avenue Henri Ravera, \\ 92225 Bagneux, France
}

(Reçu le 4 juillet; accepté le 18 novembre, 1994)

\begin{abstract}
Résumé. - Dans cette étude, nous présentons des résultats expérimentaux sur une méthode de nano-lithographie sur couche d'or (111) avec le microscope à effet tunnel. Il est possible de réaliser de véritables nano-structures (logo du laboratoire, carte, réseau ...) sur couche d'or en appliquant des pulses de tension successifs à travers la jonction tunnel. Ce travail, pour être effectué de manière contrôlée, doit impérativement être réalisé sous atmosphère contrôlée en présence de vapeur d'eau ou d'alcool. Les caractéristiques de la nano-modification (nature, taille, profondeur) sont contrôlées en changeant l'amplitude et la polarité du pulse ainsi que l'humidité relative de l'enceinte. Le plus petit trou stable qu'il est possible de former ne fait que $3 \mathrm{~nm}$ de diamètre pour une couche atomique de profondeur, ce qui correspond à une "évaporation" d'environ 100 atomes. Les structures réalisées restent stables sur une période allant au-delà de 4 jours si elles sont conservées dans l'azote pur, ce qui semblent contredire certains résultats publiés récemment sur le mouvement des atomes d'or en surface.
\end{abstract}

\begin{abstract}
Experimental results in monolayer nano-lithography on atomically flat (111) surface of gold films with scanning tunneling microscopy are presented. By applying successive voltage pulse across the tunneling gap in controlled atmosphere, the individual holes are produced in order to form different features (legend, map, test pattern or square lattice consisting of dots etc.) in nanometer scale. Our etching process has a higher success rate in nitrogen gas with the presence of water or ethanol vapor. The control of gold surface modification (feature's dimension, choice of mound or pit) was achieved by changing the pulse amplitude and polarity or the water vapour pressure. Etching feature remains stable over 4 days in dry nitrogen in contrast to some previous STM result of monoatomic step movement on gold surface.
\end{abstract}

\section{Introduction.}

Un grand effort a été fait afin d'élargir le champ d'utilisation du Microscope à Effet Tunnel (STM) au domaine de la lithographie à haute résolution [1,2]. En mettant à profit l'interaction qu'il existe entre la pointe et l'échantillon, le microscope à effet tunnel peut être utilisé comme outil de fabrication de structures nanométriques. Des travaux sur la nanofabrication ont déjà été mentionnés 
dans la littérature : par application de pulses de tension entre la pointe et l'échantillon, il est possible de créer des modifications (trous ou dépôts) de taille allant de 3 à $6 \mathrm{~nm}$ sur la couche d'or [3-9]. Lor est un candidat intéressant pour l'étude de la nano-lithographie, autant pour sa planéité à l'échelle atomique, que pour sa relative stabilité à l'air. Cependant, la plupart des études récentes faites sous-vide ou à l'air sur surface d'or, montrent la difficulté à créer des trous par cette technique de gravure. Sous ultra-vide, la probabilité d'obtenir un trou avec le STM est assez faible, tandis qu'à l'air, du fait de la contamination de surface, il n'est pas possible de contrôler la forme et la position des modifications. La grand mobilité des atomes d'or de surface implique que les modifications sur couche d'or ne se font pas à l'échelle atomique, mais plutôt à l'échelle nanométrique, contrairement au travail réalisé sur le silicium. D'autre part il est notifié que les petits trous ne sont pas stables : ils se rebouchent quelques minutes après leur création. Du fait de l'existence de ces difficultés, peu d'efforts ont été faits afin de comprendre le mécanisme de formation de ces trous, et beaucoup de questions restent posées quant à savoir ce qu'il se passe lors de la formation des nanotrous.

Dans cet article, une technique expérimentale permettant de réaliser des nanotrous sur des couches d'or, de façon reproductible et contrôlée, est présentée. Dans un premier temps, nous avons déterminé les conditions favorables pour obtenir des plateaux d'or atomiques de taille supérieure à $100 \mathrm{~nm}$. Cette étape est faite par croissance d'or sur du mica clivé, à des températures de l'ordre de $300{ }^{\circ} \mathrm{C}$. La qualité des couches obtenues ainsi que les bonnes conditions de stabilité du microscope (inférieure à $0.1 \AA$ voire même $0.01 \AA$ ) permettent d'obtenir la résolution atomique ainsi que la reconstruction $22 \times \sqrt{3}$ sur couche d'or. Nous verrons par la suite la nécessité de travailler sous atmosphère contrôlée, ainsi que l'importance de la présence de vapeur d'eau dans l'enceinte de travail. En modifiant la pression de vapeur du liquide ajouté, l'amplitude ainsi que la polarité du pulse, il est possible de contrôler la modification effectuée sur la couche d'or (taille, profondeur, nature -trou ou dépôt-). Le plus petit trou stable qu'il a été possible de réaliser a un diamètre de $30 \AA$, pour une profondeur de $2.4 \AA$, ce qui correspond à une "évaporation" d'environ 100 atomes. La mise au point de la technique de formation des nanotrous a permis de réaliser de véritables nanostructures sur couche d'or (nom de notre laboratoire, carte de France, réseau de points ...). Les modifications effectuées peuvent être conservées dans le gaz sec et observées pendant une période supérieure à 4 jours. Cette stabilité semble contredire les résultats précédemment publiés sur la diffusion des atomes d'or en surface. D'autre part, nous mentionnons pour la première fois l'existence d'un minimum du taux d'humidité relative (18\% à $22^{\circ} \mathrm{C}$ ) dans la formation du "nano-trou".

\section{Mode opératoire.}

Pour cette étude un STM disponible dans le commerce (Nanoscope III, de chez Digital Instruments, Santa Barbara, CA) a été utilisé. Ces expériences sur les modifications de surface demandent un très bon contrôle de la composition chimique de l'interface pointe-échantillon. Ainsi, plonger la tête du microscope dans un gaz neutre permet de réduire considérablement les problèmes dus à la contamination des échantillons à l'air $[10,11]$. L'unité principale du microscope est donc placée dans une boîte à gant en plexiglass, qui est ensuite purgée avec un gaz d'azote pur, pendant au moins 12 heures. L'humidité relative est alors portée au taux désiré en introduisant dans la chambre d'expérience un flux d'azote pur ainsi qu'un flux d'azote saturé en eau (cf. Fig. 1). Ce flux d'azote saturé en eau est obtenu en faisant passer, à température ambiante, de l'azote pur dans un récipient contenant de l'eau désionisée [11]. Le réglage séparé de chaque flux permet de faire varier le taux d'humidité relative continûment de 0 à $100 \%$. Le temps de stabilisation de ce taux d'humidité relative dans la boîte à gant est de l'ordre d'une demi-heure pour un flux total 


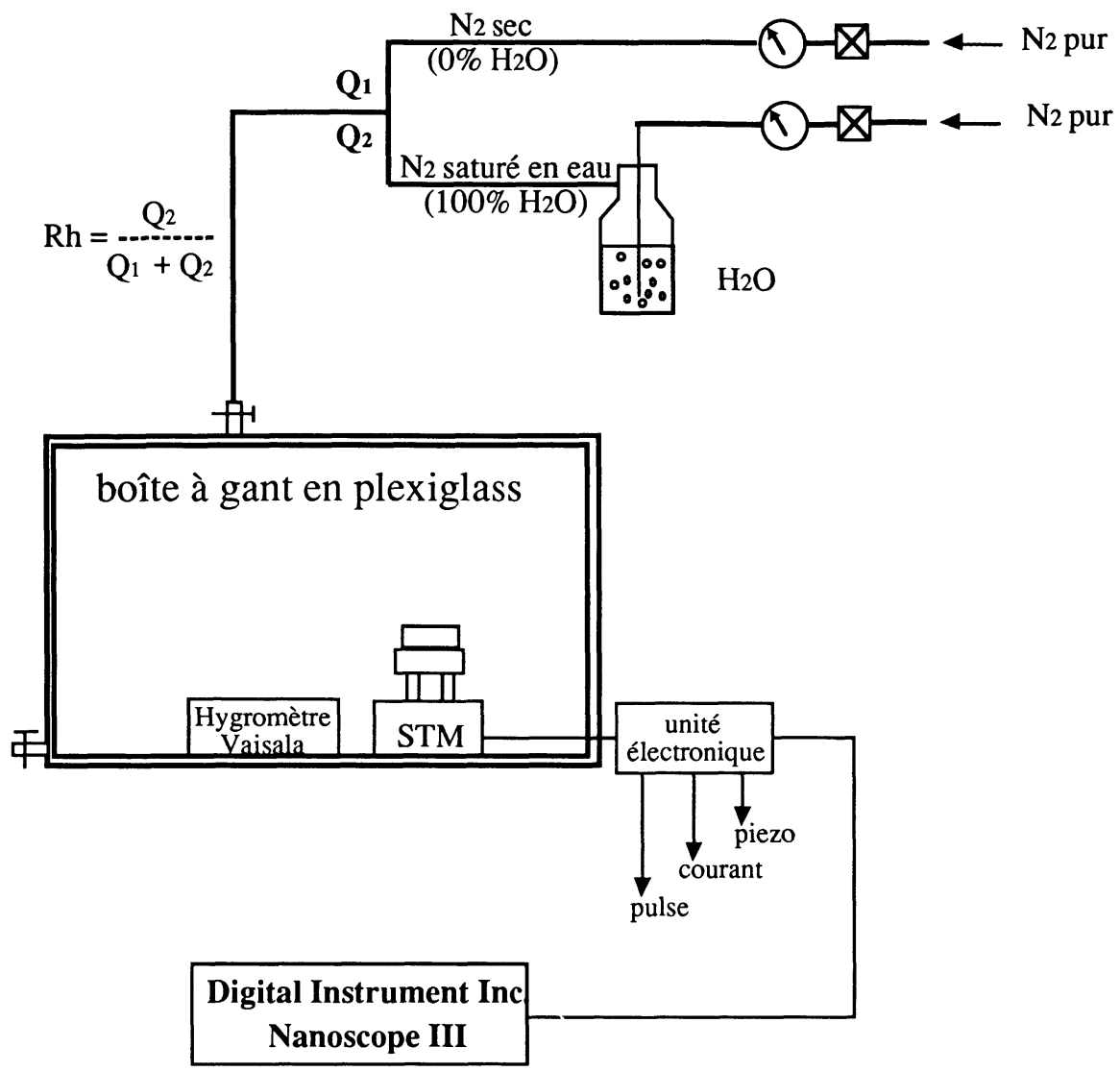

Fig. 1. - Montage expérimental permettant de travailler sous atmosphère contrôlée. [Experimental set-up in controlled atmosphere].

d'environ $4 \mathrm{l} / \mathrm{min}$. Un hygromètre digital (Vaisala, précision $1 \%$, résolution $0.1 \%$ ) est placé près de la base du microscope afin de mesurer cette humidité relative dans la chambre.

Le substrat utilisé est du mica Muscovite légèrement teinté (de couleur rouge) fourni par Digital Instruments. L'or est évaporé sur l'échantillon de mica dans une chambre à vide équipée d'une cryo-pompe ainsi que d'un sas d'introduction. Le mica est clivé immédiatement avant son introduction dans le bâti à vide. $1000 \AA$ d'or environ sont déposés à la vitesse de $1 \AA /$ s. Le substrat est placé à environ $20 \mathrm{~cm}$ de la source d'évaporation, et la pression est inférieure à $10^{-7}$ Torr. La température du substrat est maintenue à $300^{\circ} \mathrm{C}$ pendant tout le temps de l'évaporation. Le mica, composé lamellaire, adsorbe très facilement l'eau entre ses couches, un long préchauffage du substrat sous vide (au moins $24 \mathrm{~h}$ à $300^{\circ} \mathrm{C}$ ) est nécessaire pour obtenir des couches de très bonne qualité. Des analyses Auger et SIMS (Secondary Ion Mass Spectrometry) ont été faites afin d'étudier la composition de la couche sur toute son épaisseur. Aucune contamination de potassium ou de carbone n'a été détectée sur les couches réalisées. L'or se contamine dès sa mise à l'air. Après l'évaporation, l'échantillon est sorti de la chambre à vide et est immédiatement placé dans une boîte propre, le tout étant mis dans un dessiccateur. Un léger vide (inférieur à 1 Torr) est fait dans le dessiccateur puis il est rempli d'azote pur. Le temps d'exposition total à l'air des échantillons est inférieur à la demi-heure. En prenant ces précautions, les échantillons d'or dépo- 

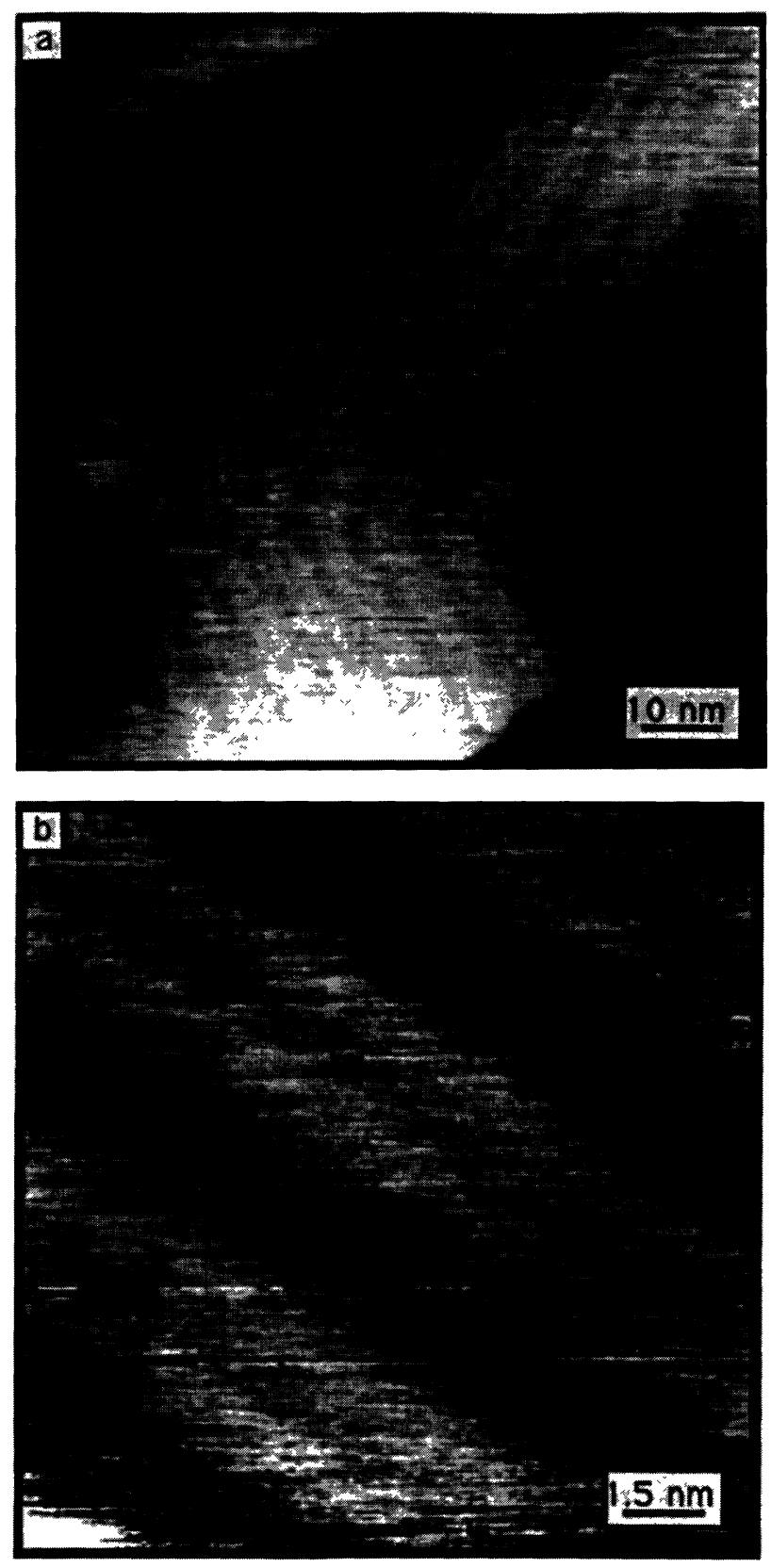

Fig. 2. - Reconstruction $22 \times \sqrt{3}$ de l'Au (111), à grande et petite échelles. Ces images ne sont pas filtrées, l'amplitude de cette reconstruction n'est que d'environ $0.1 \AA$.

$[22 \times \sqrt{3}$ surface reconstruction of gold (111). Those images are not filtered, the corrugation of the reconstruction is only $0.1 \AA$ ].

sés sur du mica peuvent être gardés environ 10 jours sans contamination gênante pour l'étude de la nano-écriture.

Occasionnellement, sous azote ou sous argon hydrogéné la reconstruction $22 \times \sqrt{3}$, d'amplitude 


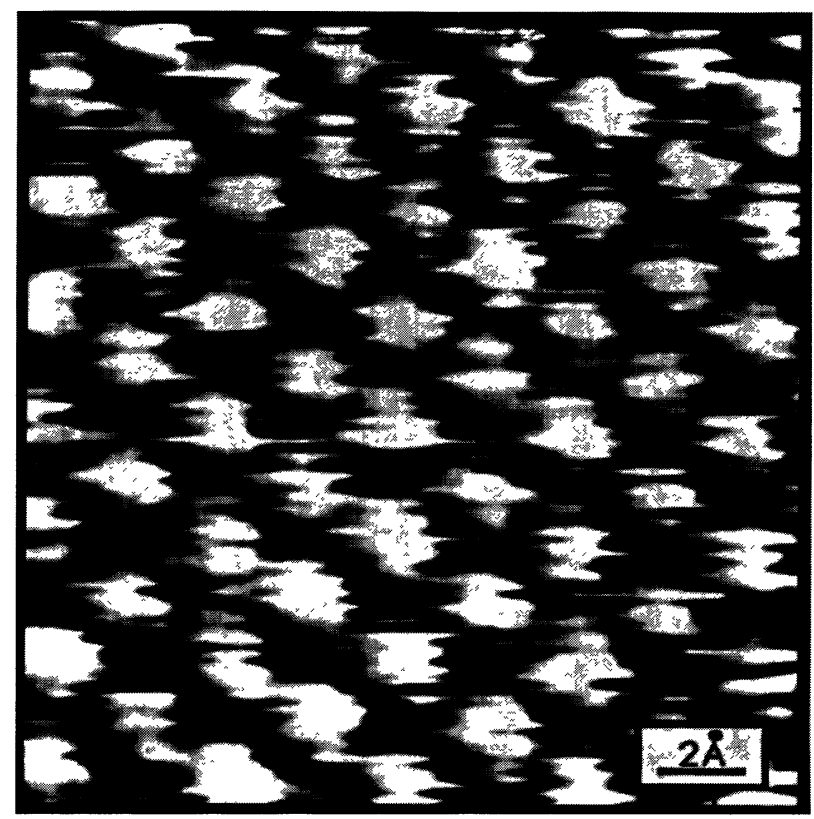

Fig. 3. - Résolution atomique de l'Au (111). Le paramètre de maille du réseau est de $2.8 \AA ̊$. [Atomic resolution STM image of $\mathrm{Au}(111)$ single crystal. The gold interatomic distance is $2.8 \AA$ ].

$0.1 \AA$, est observée (cf. Figs. $2 \mathrm{a}$ et $2 \mathrm{~b}$ ) ainsi que la résolution atomique sur le réseau hexagonal de l'Au (111) (cf. Fig. 3), prouvant la qualité des couches. Ces observations ne sont pas une condition nécessaire pour la formation de trou à la surface de l'or. Notre critère pour caractériser la propreté des échantillons d'or est fondé sur l'observation de bords de marches droits (non sinueux) ainsi que de terrasses nettes (à l'échelle de $1 \mathrm{~nm}$ en $z$ ). Un exemple de plateau de bonne qualité est représenté sur la figure 4. Cette image a été réalisée en mode courant constant, l'échantillon étant sous atmosphère contrôlée. Aucun filtre n'est utilisé pour cette image, les conditions de travail sont de $100 \mathrm{mV}$ et $1 \mathrm{nA}$. La mesure de la hauteur de la barrière tunnel est également un critère de propreté : si le courant tunnel est défini par $I=A V \exp (-2 B \sqrt{\phi} d)$, une valeur de $\phi$ au dessus de $0.5 \mathrm{eV}$ nous convient. Sur des surfaces sales, bien souvent le courant tunnel est peu stable et montre une faible dépendance de la distance pointe - échantillon, signe d'une faible valeur de $\phi$. Les modifications de surface sur des couches d'or "sales" sont possibles mais incontrôlables, la réalisation de la nano-écriture est alors très difficile.

Les nano-modifications sont réalisées en immobilisant la pointe au dessus de l'échantillon, à une distance imposée par les conditions standard de balayage $(100 \mathrm{mV}, 1 \mathrm{nA})$, puis en appliquant un pulse de tension entre la pointe et l'échantillon. La largeur du pulse varie de $1 \mathrm{~ms}$ à $2 \mathrm{~s}$. Lasservissement fait sur le courant permet de le maintenir à la valeur imposée précédemment (1 nA).

Rabe et al. [12] ont suggéré que la présence de vapeur d'eau était une condition nécessaire pour former des trous dans le graphite. Il se trouve que nous obtenons la même condition pour la gravure sur couche d'or. Nous avons également effectué quelques expériences avec des vapeurs d'éthanol : il semblerait que la nano-écriture soit également réalisable avec ce composé chimique. Cependant une étude plus précise a été conduite avec l'eau. Le taux d'humidité relative dans la boîte à gant a été varié de 0 à $50 \%$ environ. Le seuil de tension pour former un trou d'une 


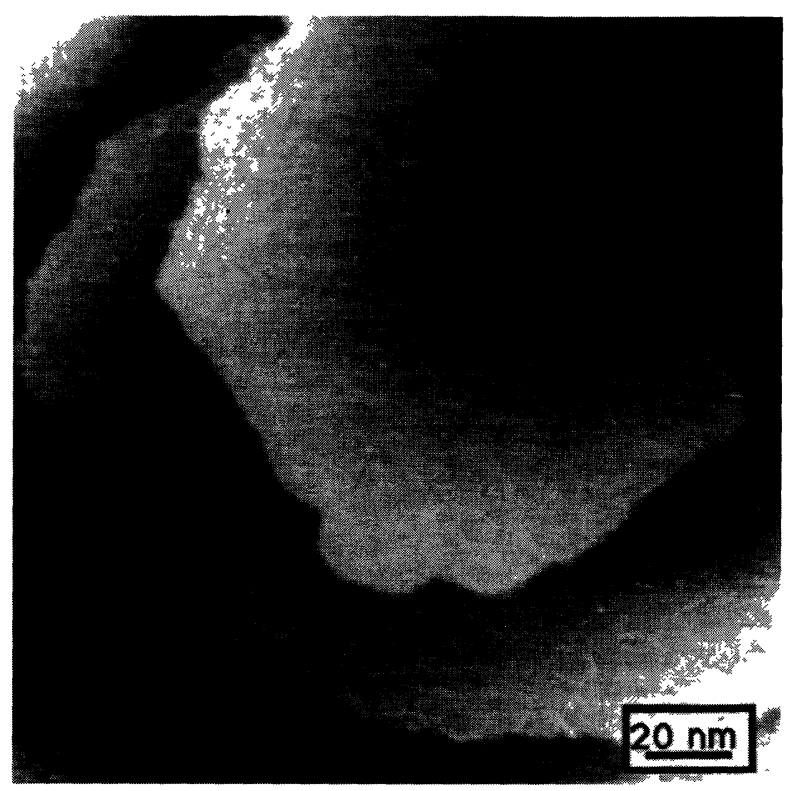

Fig. 4. - Image STM d'un plateau sur une couche d'Au (111). Cette image a été réalisée en mode courant constant, l'échantillon étant sous atmosphère contrôlée. Aucun filtre n'est utilisé pour cette image, les conditions de travail sont de $100 \mathrm{mV}$ et $1 \mathrm{nA}$. En $Z$ l'échelle est de $1 \mathrm{~nm}$.

[STM image in controlled atmosphere of an atomically flat gold plate. Experimental conditions for this image: $V=100 \mathrm{mV}, I=1 \mathrm{nA}$, image in constant current mode].

monocouche de profondeur est défini sur la figure 5. Cette figure représente la dépendance de la profondeur des trous gravés (symboles blancs) et la hauteur des dépôts réalisés (symboles noirs) en fonction de la tension du pulse appliquée, pour différents taux d'humidité relative. La ligne en pointillés symbolise un trou d'une monocouche de profondeur. Le seuil de tension est alors défini comme l'intersection de cette droite avec la courbe représentant la profondeur du trou gravé en fonction de l'intensité appliquée. Il peut en effet être défini comme étant la plus petite tension qu'il faut appliquer pour former un trou d'une monocouche. Sous ce seuil on peut observer une légère marque sur la couche, de profondeur bien inférieure à la monocouche. Ce signe précurseur du trou d'une monocouche peut être une déformation inélastique de la surface (111). Au delà de la tension seuil, il faut noter la quantification de la profondeur des trous créés, en effet c'est un nombre entier de fois la profondeur d'une monocouche $(2.35 \AA)$. Il est impossible de faire un trou en appliquant une tension négative (l'échantillon est alors négatif).

Lexistence d'une humidité relative critique $\left(R h_{\mathrm{c}}\right)$ apparaît clairement sur la figure 6 . Nous avons, sur cette courbe, reporté le seuil de tension en fonction de l'humidité relative pour quatre échantillons différents. Les symboles blancs représentent toujours les trous, tandis que les noirs représentent les dépôts. Nous définissons le taux d'humidité relative critique comme étant le saut du seuil de tension apparaissant pour une humidité de $18 \%$ environ. En dessous de ce taux d'humidité, il est impossible de former des trous de façon reproductible, ce sont essentiellement des dépôts qui sont créés à une tension comprise entre 3.3 et $3.7 \mathrm{~V}$. Au dessus de $R h_{\mathrm{c}}$, la formation des trous a lieu pour une tension de l'ordre de $2.7 \mathrm{~V}$. Il faut noter la reproductibilité des résultats d'un échantillon à l'autre, en effet le taux d'humidité relative critique est le même pour tous les 


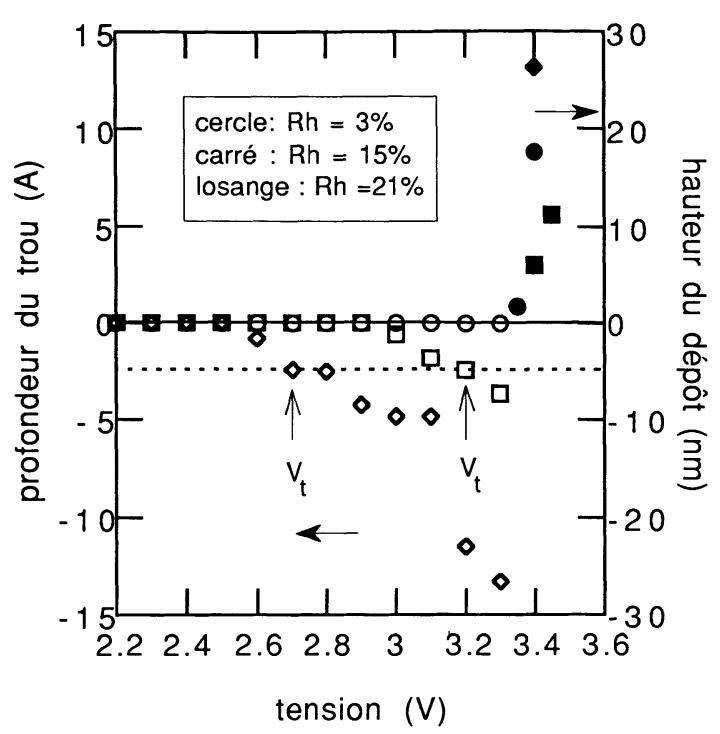

Fig. 5. - Définition du seuil de tension, cette figure représente la dépendance de la profondeur des trous gravés (ou la hauteur des dépôts réalisés) en fonction de la tension du pulse appliqué, pour différents taux d'humidité relative. La ligne en pointillés indique une profondeur d'une monocouche. Les symboles blancs représentent les trous, tandis que les noirs représentent les dépôts. Le seuil de tension $(V t)$ est défini comme étant le minimum de tension qu'il faut appliquer pour obtenir un trou d'une monocouche de profondeur. [Threshold voltage definition. This figure represents the dependence of the etched pit depth (or deposited mound height) on the pulse voltage at different relative humidity. Discontinuous line indicates the monolayer depth in (111) direction of gold. White symbols represent the pit and black ones represent the mound. The bias threshold voltage $(V t)$ is defined as that necessary to produce a monolayer hole on the surface of gold which could be subsequently observed by STM].

échantillons. Le trou créé, au delà de $R h_{\mathrm{c}}$, peut avoir une forme arbitraire juste après le pulse, néanmoins il prendra une forme circulaire dans les quelques secondes qui suivent sa création. Le plus petit trou stable qu'il a été possible d'obtenir ne fait que $30 \AA$ de diamètre et $2.4 \AA$ de profondeur, ce qui correspond environ à une "disparition" d'une centaine d'atomes. Le devenir de ces atomes manquant est inconnu, mais la propreté des contours du trou montre clairement que ces atomes se trouvent loin de la modification.

Les pointes utilisées sont fournies par Digital Instruments, elles sont en platine-iridium. La résistance de ces pointes a été démontrée par leur capacité à graver la couche d'or et à obtenir ensuite des images des nanostructures pendant plusieurs jours. Une légère dégradation de la qualité des images et des trous formés au cours du temps est tout de même observée. Il semble que la résolution atomique ne peut pas être obtenue après la formation d'une modification, ce qui peut permettre d'interpréter la modification comme créant une contamination sur la pointe. Ce peut être dû à l'attaque progressive de la pointe qui a lieu lors des dépôts augmentant la surface effective du bout de la pointe. Cependant une méthode de "nettoyage" [13,14] peut être utilisée : un pulse de $3.7-4 \mathrm{~V}$ (juste au dessus de la tension de formation des dépôts) est appliqué afin de nettoyer la pointe après une centaine de trous fabriqués. 


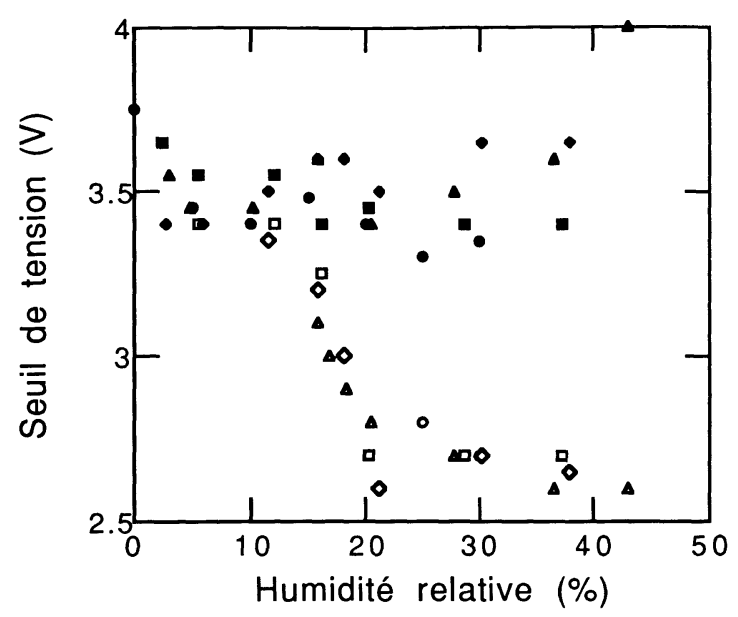

Fig. 6. - Relation entre le seuil de tension et le taux d'humidité relative dans la chambre d'expérience pour différents échantillons. L'existence d'un taux d'humidité critique apparaît clairement pour une humidité relative de l'ordre de $18 \%$. Les symboles blancs représentent les trous et les noirs les dépôts.

[The relationship between threshold voltage $(V t)$ and relative humidity for four samples. The existence of a critical humidity value $\left(R h_{\mathrm{c}}\right)$ at $18 \%$ is clearly presented. White symbols represent the pit and black ones represent the mound].

\section{Nano-écriture sur couche d'or.}

L'humidité de l'enceinte est fixée à $20 \%$ afin d'être dans les meilleures conditions de formation de trous, cette condition est imposée par l'étude de la figure 6. Il faut cependant éviter d'exposer l'échantillon à une humidité élevée pendant trop longtemps, car sa surface devient "sale" et la réalisation de la nano-écriture y est incontrôlable. Sur un échantillon de bonne qualité, en contrôlant la position de la pointe et donc de la modification, des nanostructures ayant une forme prédéterminée peuvent être créées. La première structure présentée en figure 7 est un "L" qui a été créé par environ 7 pulses successifs. Il faut remarquer la quasi-planéité du fond de la structure, qui apparaît sur les sections horizontales et verticales de la figure. Chaque trou est formé par application d'un pulse de tension à travers la jonction tunnel, l'échantillon étant plongé dans une atmosphère contrôlée $\left(R h 20 \%, 22^{\circ} \mathrm{C}\right)$. Il faut également remarquer la profondeur de cette écriture qui n'est que d'une couche atomique, soit $2.35 \AA$.

Près de 150 trous individuels ont été créés afin d'enregistrer le message de la figure 8 sur couche d'or. Les lettres ont une hauteur totale d'environ $300 \AA$ pour une largeur de l'ordre de $250 \AA$. Elles sont formées de traits de $50 \AA$ de largeur et de seulement $2.35 \AA$ de profondeur. Un taux de reproductibilité proche de $100 \%$ est indispensable pour réaliser une telle nano-structure, en effet la moindre petite erreur (nanotrou mal positionné, trop profond ou dépôt à la place du trou) implique une perte irrémédiable de la nano-structure.

La carte de France de la figure 9 est réalisée au 1/10.000.000.000.000 ième. Le nombre d'atomes “évaporés" n'est que de 32.000. Enfin sur la figure 10 un réseau de $4 \times 4$ points est présenté. Cette structure montre la précision du positionnement des motifs qu'il est possible d'obtenir. En effet cette précision peut être évaluée à $\pm 1 \mathrm{~nm}$. Il faut également remarquer la forme et la taille relativement reproductibles des nanotrous. 

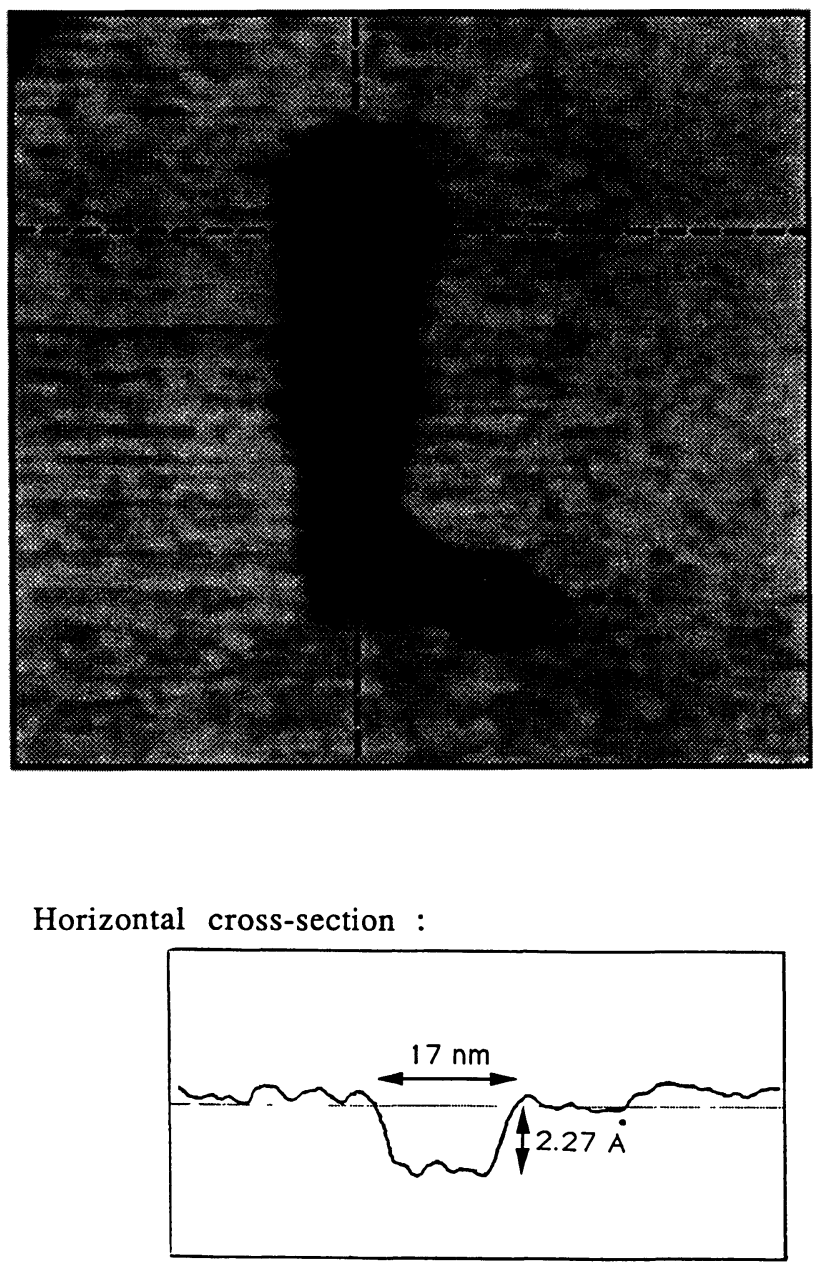

Vertical cross-section :

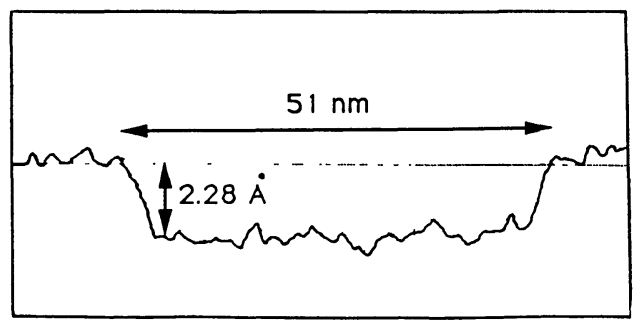

Fig. 7. - Nano-écriture sur couche d'or. Analyse de sections horizontale et verticale de la lettre "L". La profondeur de cette lettre est quasiment uniforme est égale à une monocouche. Aucun atome résiduel n'est remarqué autour de la lettre.

[Nano-writing on gold surface. Cross-section analysis of writing letter $\mathrm{L}$ in two directions reveals the nature of monolayer nano-writing: the depth of the etching lines is quite uniform and equals to the monoatomic step height. No debris around the through was found]. 


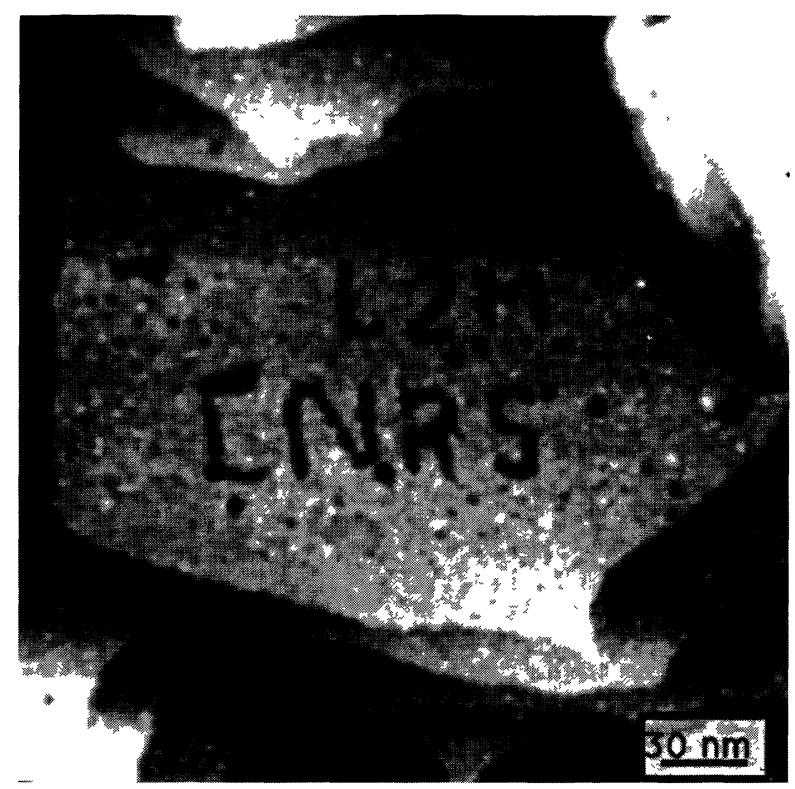

Fig. 8. - Nanostructure 1 sur couche d'or. Nom du laboratoire. Près de 150 trous individuels ont été créés afin d'enregistrer ce message sur couche d'or. Les lettres ont une hauteur totale d'environ $300 \AA$ pour une largeur de l'ordre de $250 \AA$. Elles sont formées de traits de $50 \AA$ de largeur et de seulement $2.35 \AA$ de profondeur.

[Nanostructure 1 on gold surface. Nearly 150 individual holes were produced to record the legend "L2M CNRS" on the gold surface. The letters are about $300 \AA$ tall and $250 \AA$ wide and are made up of lines of $50 \AA$ in width and $2.4 \AA$ in depth (monolayer writing). The lines are formed by the coalescence of the neighbour holes due to the fast surface diffusion].

\section{Diffusion de surface et stabilité des nano-modifications créées.}

Après avoir réalisé les motifs, la boîte à gant est à nouveau purgée avec, cette fois, de l'azote pur. Les structures peuvent alors être conservées plusieurs jours dans cet environnement. En effet une nanomodification a pu être conservée pendant 4 jours, malgré une légère dégradation de l'image, qui peut être due soit à l'altération de la pointe ou à celle de la structure elle-même (cf. Fig. 11). Il est possible que des structures d'une monocouche de profondeur soit plus stables que des motifs formés sur plusieurs couches.

La disparition progressive des nanomodifications réalisées est un phénomène qui a été largement reporté dans la littérature. La taille de ces nano-structures observées par STM, décroît en fonction du temps sur des périodes allant de quelques minutes à quelques heures.

Ce phénomène a été interprété comme étant dû à la diffusion de surface des atomes d'or. Le coefficient de cette "auto-diffusion" est de l'ordre de $10^{-14}$ à $10^{-16} \mathrm{~cm}^{2} / \mathrm{s}$ [14, 15]. Ce type de diffusion sera nommé la diffusion rapide des atomes d'or. Lécriture des lettres est un exemple de ce modèle : deux pulses successifs sont effectués créant ainsi deux trous distants de quelques nanomètres $(<3 \mathrm{~nm})$, au cours du temps ces deux trous vont coalescer pour former un unique trou ellipsoïdal dont le petit axe est proche du diamètre des trous originalement formés (voir Fig. 12). Ce procédé a lieu quelques minutes après la formation des simples trous. Un calcul approximatif, montre que le nombre d'atomes manquant avant et après coalescence des trous est le même. Ainsi 


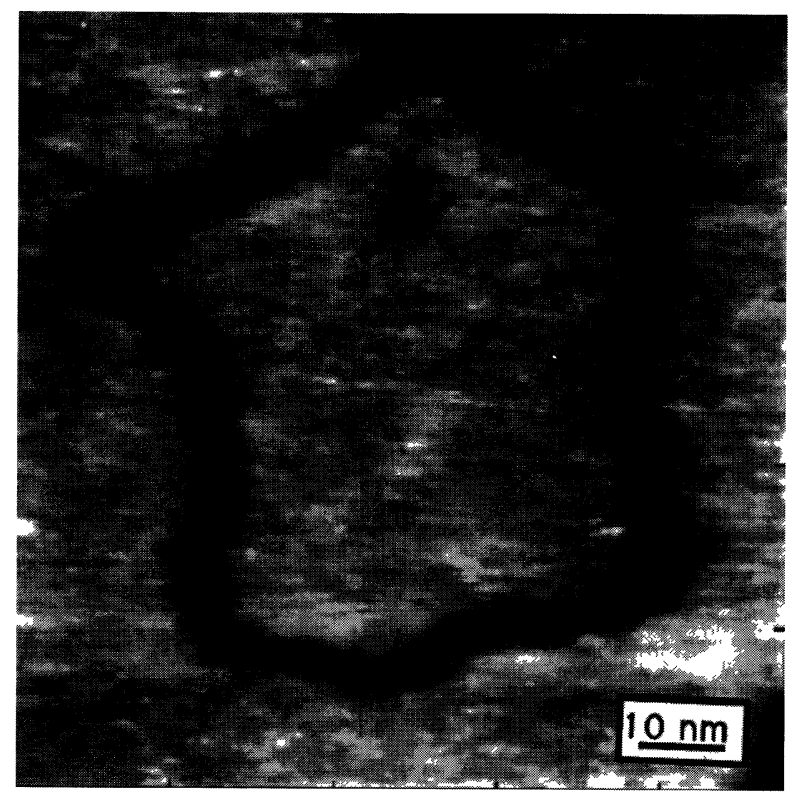

Fig. 9. - Nanostructure 2. Carte de la France au 1/10.000.000.000.000. Seuls 32.000 atomes ont été “évaporés".

[Nanostucture 2. The map of France (1/10.000.000.000.000). Only 32000 atoms have been "evaporated"].

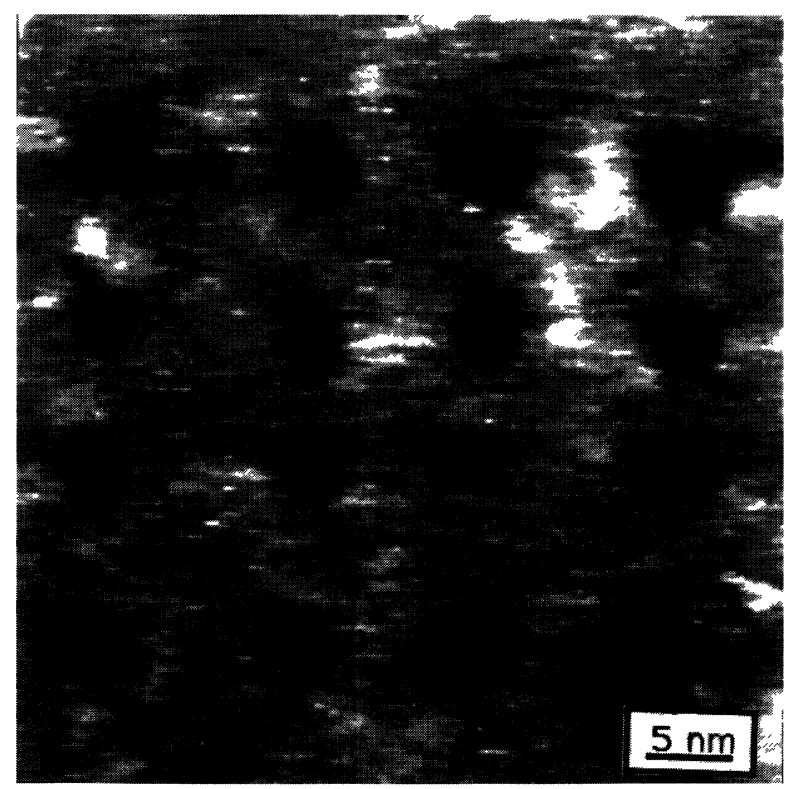

Fig. 10. - Nanostructure 3. Réseau de 16 points. La précision du positionnement des nano-trous peut être évaluée à $\pm 1 \mathrm{~nm}$.

[Nano-structure 3. Lattice with $4 \times 4$ dots. The precision of the nano-writing is supposed to be $\pm 1 \mathrm{~nm}$ ]. 


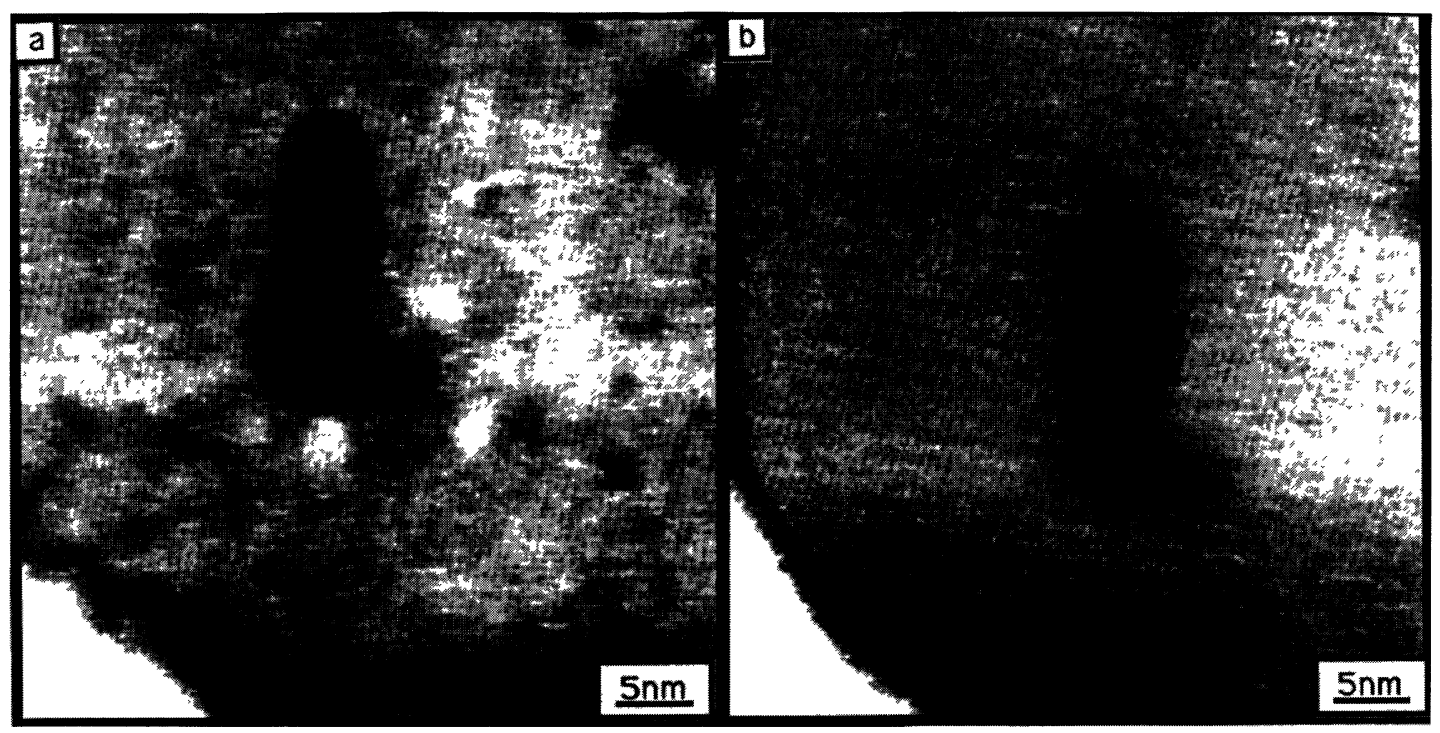

Fig. 11. - Stabilité de la nano-écriture. Evolution au cours du temps de la lettre "L". Ces images ont été prises a deux instants différents : a) cinq minutes après l'écriture. b) trois jours plus tard. Ce résultat montre la relative stabilité au cours du temps des nano-structures.

[Nano-writing stability. Time evolution of letter "L". Images were taken at different times: a) five minutes after writing. b) three days later. The results show that the time evolution is less important].

chaque trou coalescera avec ses voisins de part et d'autre, afin de créer des lignes permettant la réalisation de formes continues. Il est possible que le mouvement de coalescence observé soit dû à la diffusion de surface des atomes d'or, s'arrangeant alors dans une configuration stable. Par ailleurs, la contrainte créée par la surface perturbée peut être la cause de cette diffusion. La fusion des trous voisins est énergétiquement favorable dans la mesure où la longueur des parois est réduite par un facteur $\sqrt{2}$, et par la même réduit l'énergie totale de surface. La vitesse de diffusion des atomes dans le cas de ce mouvement rapide varie de 0.1 à $4 \AA$ $/$ s, valeurs qui sont en accord avec le résultat de Jaklevic [15].

Cependant, il apparaît dans notre étude un fait nouveau par rapport aux travaux rapportés jusqu'à présent : aucun mouvement ou comblement de structures préexistantes en surface (telles que des petits trous même si leur dimension est inférieure à $10 \mathrm{~nm}$ ) ne sont observés. Dans la théorie de l'auto-diffusion, ces structures devraient disparaître en environ $100 \mathrm{~s}\left(t=L^{2} / D, L=100 \AA\right.$ et $D=10^{-14}$ à $10^{-16} \mathrm{~cm}^{2} / \mathrm{s}$ ). Peale et Cooper [8] ont remarqués que, dans des conditions d'ultravide, des nanostructures constituées d'adatomes restent inchangées sur une longue période de temps. La décroissance en taille de ces structures peut être initialisée par l'ajout de certains adsorbats sur la surface (111) de l'or. Ils interprètent alors la décroissance de la taille des îlots comme étant un régime de transfert de masse, assisté par adsorption de certains adatomes (par exemple $\mathrm{Cl}$...). Il apparaît alors un coefficient de diffusion sous ultra-vide qui serait 5 ordres de grandeur plus faible que celui de Jaklevic. L'observation de la figure 2 sous gaz d'azote pur appuierait cette hypothèse : les lettres sont stables au delà de 4 jours, ce qui signifie que la vitesse de diffusion est inférieure à $1 \AA / \mathrm{h}$ bien que l'expérience soit effectuée sous azote et non sous ultra-vide. Cette vitesse est 1000 fois plus faible que celle observée dans les premiers instants de formation des trous. Il est donc nécessaire de distinguer deux types de décroissance dans le procédé de nano-écriture 


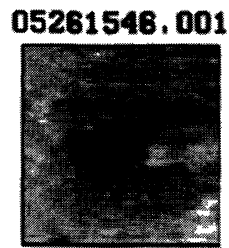

05281549.001

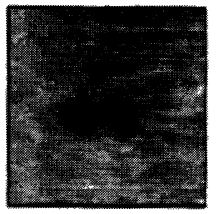

05261551,002

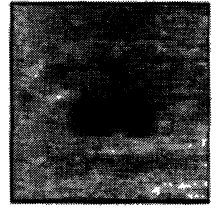

05261558.002

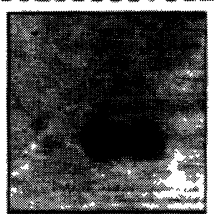

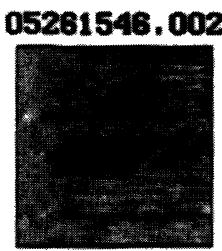

05281549.002
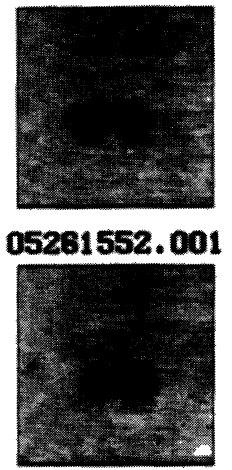

05261557.001

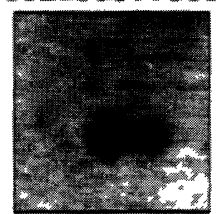

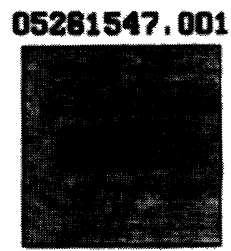

05281548.003
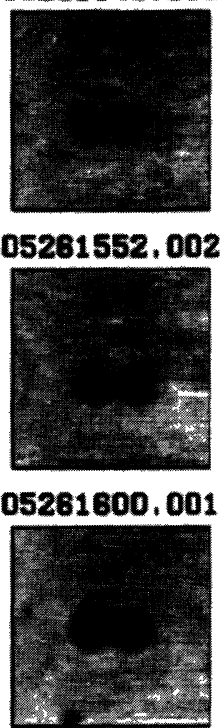

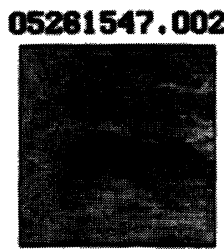

05281550.001
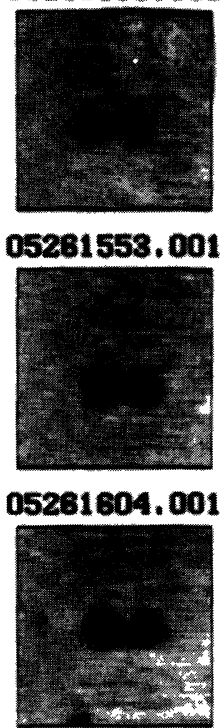

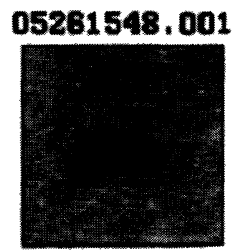

05261548.002
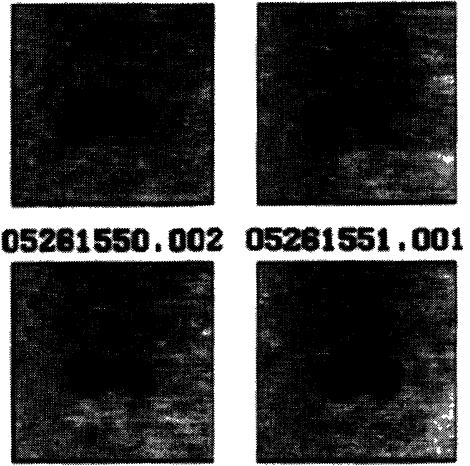

05261551.001
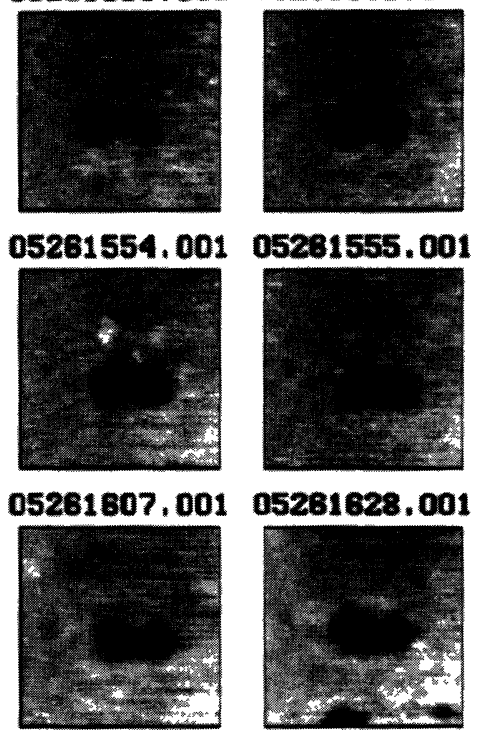
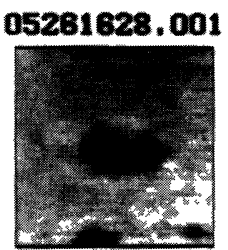

Fig. 12. - Cette série d' images a été enregistrée afin d'observer la coalescence de deux trous distincts. Deux pulses successifs sont effectués créant ainsi deux trous distants d'environ $2 \mathrm{~nm}$, au cours du temps ces deux trous vont coalescer pour former un unique trou ellipsoïdal dont le petit axe est proche du diamètre des trous originalement formés. Le numéro situé au dessus des images se lit de la façon suivante : mois/jour/heure/minute.

[Coalescence of two holes. This succession of images has been recorded in order to observe the coalescence of two neighbour pits. Two successive pulses have been done, creating two neighbour pits $(<3 \mathrm{~nm}$ in distance). A few minutes later the two pits will unite to become a larger "hole" that has an elliptical shape with a short axis close to the original pit's diameter. The number, above images, means: month/day/hour/minutes].

sur couche d'or : une décroissance rapide ayant un coefficient de diffusion de l'ordre de $10^{-14}$ à $10^{-16} \mathrm{~cm}^{2} / \mathrm{s}$ (diffusion de surface de Jaklevic) et une décroissance beaucoup plus lente avec un coefficient de diffusion de l'ordre de $10^{-22} \mathrm{~cm}^{2} / \mathrm{s}$ (transfert de masse de Peale). Lors de la création d'un trou nanométrique, la configuration originale stable (arrangement atomique) est perturbée, il se crée un réarrangement atomique de façon à minimiser l'énergie libre du système. Du fait de la faible valeur de la barrière énergétique qu'il existe entre les deux positions métastables, les atomes se réarrangent rapidement afin de trouver une position énergétiquement stable. Puis apparaît ensuite un second mouvement beaucoup plus lent, qui permet aux atomes d'atteindre la seconde configuration d'énergie plus basse. D'une façon ou d'une autre, la relaxation du champ de contrainte doit jouer un rôle important dans la diffusion rapide. La coexistence de deux diffusions dans un même système n'est pas un phénomène nouveau : un grand nombre de systèmes complexes, loin de leur position d'équilibre, peuvent avoir plus d'un mécanisme de relaxation (par 
exemple les verres de spin). Ces mécanismes doivent être étudiés séparément. Le mécanisme détaillé du transfert des atomes vers ou en dehors des bords de marche doit être clarifié dans le futur. Du fait de l'existence d'un fort champ électrique et d'une haute densité de courant sous la pointe du STM, la présence de la pointe doit avoir une grande influence sur le procédé de diffusion [16]. Dans certains cas, la détérioration des motifs créés provient clairement de l'interaction qu'il existe entre la pointe et l'échantillon, interaction qui ne doit pas être négligée. Il est évident que ce problème sera crucial pour la mise au point d'une application potentielle de la nano-écriture.

\section{Conclusion.}

Dans ce travail une technique qui permet de réaliser des nanostructures par lithographie, grâce au STM, a été développée. Pour la première fois, une méthode reproductible et contrôlée de nanogravure sur couche d'or est présentée. En effet, il est possible de contrôler la profondeur des modifications à une couche atomique près en variant l'intensité du pulse de tension. Nous pouvons alors en pilotant la pointe du microscope réaliser de véritables nanostructures par création de multiples nanotrous contigus. La condition indispensable à la nanoécriture est le travail sous atmosphère contrôlée en présence de vapeur d'eau (ou d'éthanol) : un taux minimum de $20 \%$ d'humidité relative est nécessaire. D'autre part les motifs créés sont stables au delà de 4 jours. Une étude sur l'origine du mécanisme de la nano-écriture et sur la variation de la hauteur de la barrière de l'effet tunnel dans différentes humidités relatives, est en cours. Un futur travail pour la nanoécriture serait la formation de structures à l'échelle nanométrique afin d'étudier les propriétés physiques de telles structures (résolution de structures électroniques dans l'espace réel, ondes stationnaires des électrons sur surface d'or ...) [17].

\section{Remerciements.}

Nous remercions Mme H. Launois ainsi que Mr S. Gauthier pour leurs discussions enrichissantes ainsi que pour leurs encouragements. Nous tenons également à remercier Mrs C. Mayeux, J. Rosiu, J. Bourneix et C. David pour leur aide précieuse.

\section{Bibliographie}

[1] Sedd G.M. and Russell P.E., "The scanning tunneling microscope as a tool for nanofabrication”, Nanotechnology 4 (1990) 67-80.

[2] Wiesendanger R., "Contributions of scanning probe microscopy and spectroscopy to the investigation and fabrication of nanometer-scale structures", J. Vac. Sci. Techol. B12 (1994) 515-529.

[3] Emch R., Nogami J., Dovek M.M. , Lang C.A., Quate C.F., "Characterization of gold surface for use as substrates in scanning tunneling microscopy studies”, J. Appl. Phys. 65 (1989) 79-84.

[4] Guo C.X., Thomson D.J., "Material transfer between metallic tips and surface in the STM", Ultramicroscopy 42-44 (1992) 1452-1458.

[5] Hasegawa Y., Avouris Ph., "Manipulation of the reconstruction of the Au(111) surface with the STM", Science 258 (1992) 1763-1765.

[6] Li Y.Z., Vazquez L., Piner R., Andres R.P., Reifenberger R., "Writing nanometer-scale symbols in gold using the scanning tunneling microscope", Appl. Phys. Lett. 54 (1989) 1424-1426, and Appl. Phys. Lett 55 (1989) 2366-2367. 
[7] Mamin H.J., Guethner P.H., Rugar D., "Atomic emission from a gold scanning tunneling microscope tip”, Phys. Rev. Lett. 65 (1990) 2418-2420.

[8] Peale D.R., Cooper B.H., "Adsorbate-promoted mass flow on the gold (111) surface observed by scanning tunneling microscopy", J. Vac. Sci. Technol. A10 (1992) 2210-2215.

[9] Schneir J. and Hansma P.K., "Scanning tunneling microscopy and lithography of solid surface covered with nonpolar liquids", Langmuir 3 (1987) 1025-1027.

[10] Roberts C.J., Hoffmann-Millack B., Steer W.S., "Observation of a gold surface using scanning tunneling microscopy in an inert atmosphere and the effects of water adsorption", J. Vac. Sci. Technol. B9 (1991) 841-844.

[11] Thundat T., Zheng X.Y., Chen G.Y., Warmack R.J., "Role of relative humidity in atomic force microscopy imaging”, Surf. Sci. Lett. 294 (1993) L939-L943.

[12] Rabe J.P., Buchholz S., Ritcey A.M., "Reactive graphite etch and the structure of an adsorbed organic monolayer-a scanning tunneling microscopy study", J. Vac. Sci. Technol. A8 (1990) 679-683.

[13] Mamin H.J., Chiang S., Birk P.H., Guethner P.H., Rugar D., "Gold deposition from a scanning tunneling microscope tip”, J. Vac. Sci. Technol. B9 (1991) 1398-1402.

[14] Emch R., Nogami J., Dovek M.M., Lang C.A., Quate C.F., "Characterization of gold surface for use as substrates in scanning tunneling microscopy studies”, J. Appl. Phys. 65 (1989) 79-84.

[15] Jaklevic R.C. and Elie L., "Scanning tunneling microscope observation of surface diffusion on an atomic scale : Au on Au(111)", Phys. Rev. Lett. 60 (1988) 120-123.

[16] Mo Y.W., "Direct determination of surface diffusion by displacement measurement with scanning tunneling microscopy”, Phys. Rev. Lett. 71 (1993) 2923-2926.

[17] Lebreton C. et Wang Z.Z., à paraître. Récemment, une étude préliminaire, en utilisant le microscope à force atomique en tapping mode, confirme les résultats obtenus en STM sur la création des nanostructures. 